\title{
Changes of anisotropy of dilatative and optical properties of DGN crystal at ferroelectric phase transition
}

\author{
B.V. Andriyevsky ${ }^{1}$, M.O. Romanyuk ${ }^{2}$, Yu.A. Dumka ${ }^{2}$ \\ ${ }^{1}$ Faculty of Electronics, Technical University of Koszalin, 17 Partyzantów Str., PL-75-411, Koszalin, Poland \\ E-mail: bandri@tu.koszalin.pl \\ ${ }^{2}$ The Ivan Franko National University of L'viv, 8 Kyryla i Mefodiya Str., 79005 L'viv, Ukraine
}

\begin{abstract}
Temperature dependences of the linear dimension $L(T)$ and the optical path difference $D(T)$ of the ferroelectric diglycine nitrate crystals, $\left(\mathrm{NH}_{2} \mathrm{CH}_{2} \mathrm{COOH}\right)_{2} \cdot \mathrm{HNO}_{3}$, have been measured for three principal directions of optical indicatrix in the range of $130 \div 295 \mathrm{~K}$ including the phase transition point $T_{\mathrm{c}}=206 \mathrm{~K}$. Temperature dependences of the refractivity $n(T)-1$, refraction $R(T)$, and degrees of dilatative and optical anisotropies, $A_{\alpha}(T)$ and $A_{n-1}(T)$ have been calculated on the basis of the experimental dependences $L(T)$ and $D(T)$.

Minima of the temperature dependences of spontaneous electrostriction coefficients $Q_{i j}{ }^{(s)}(T)$, anisotropy degrees of dilatative $\left(A_{\alpha}\right)$ and refractive $\left(A_{n-1}\right)$ characteristics close to $T_{\mathrm{c}}$ temperature show the relative disordering of DGN crystal in the temperature range near $T_{\mathrm{c}}$. The temperature dependence of refraction $R(T)$ of the crystal testifies the increase of dipole-dipole interaction with decreasing temperature in the wide range $295 \div 130 \mathrm{~K}$.
\end{abstract}

Keywords: ferroelectric crystals, phase transition, anisotropy of physical properties

Paper received 06.11.02; accepted for publication 18.03.03.

\section{Introduction}

The crystal of diglycine nitrate $(\mathrm{DGN}),\left(\mathrm{NH}_{2} \mathrm{CH}_{2} \mathrm{COOH}\right)_{2}$. $\mathrm{HNO}_{3}$, is ferroelectric below the phase transition temperature $T_{c}=206 \mathrm{~K}$ belonging to the space group $P a$, and above this temperature it becomes paraelectric with the center symmetry space group $P 2_{1} / a[1,2]$. It was suggested from the dielectric and specific heat measurements that the phase transition of this crystal is of order-disorder type [1]. Structural arrangement of two glycine molecules as well as a hydrogen bond between them and a mechanism of the phase transition were found to be fairly similar to the well-known triglycine sulphate crystals (TGS). The spontaneous polarization vector $P_{s}$ of DGN crystal lies in the symmetry plane (101) [2] and is equal to $1.4 \cdot 10^{-2} \mathrm{C} / \mathrm{m}^{2}$ at $123 \mathrm{~K}$ [1].

It was shown in the investigation of optical properties of DGN crystal [3] that $b$-axis of the crystal is perpendicular to the symmetry plane and coincides with $n_{m^{-}}$ direction of the optical indicatrix. The angle between the spontaneous polarization vector $P_{\mathrm{s}}$ and $n_{p}$-axis is equal to $17.2^{\circ} \pm 0.5^{\circ}$. Principal magnitudes of birefringence of DGN for the wavelength $\lambda=632.8 \mathrm{~nm}$ are the following, $\Delta n_{g}=n_{m}-n_{p}=0.079, \Delta n_{m}=n_{g}-n_{p}=0.141, \Delta n_{p}=$ $=n_{g}-n_{m}=0.070$. The crystal is transparent in the spectral range of $245 \div 1900 \mathrm{~nm}$.
The aim of present investigation was to study the peculiarities of temperature behavior of degree of anisotropy of physical properties of DGN crystal in the range including phase transition point $T_{c}$.

\section{Experimental}

The samples of DGN single crystal were grown by the slow evaporation method. Optically transparent samples of DGN crystal were prepared in the form of rectangular blocks cut along the principal directions of optical indicatrix with typical dimensions of about $5 \div 10 \mathrm{~mm}$.

The thermal linear expansion of DGN crystal was measured using the quartz dilatometer by the electric capacitor method [4]. The rate of temperature change of the samples during cooling run was $1 \mathrm{~K} / \mathrm{min}$. The sensitivity of dilatometer $\delta L$ being a function of distance between capacitor plates was equal to $\delta L \approx 5 \mathrm{~nm}$ in our case. This corresponds to the relative error $\delta(\delta L / L) \leq 10^{-6}$ of the elongation $\delta L / L$.

The dispersion of refractive index $n(\omega)$ of DGN crystals was measured with an accuracy of $\delta n=2 \cdot 10^{-4}$ by the immersion method using the refractive index dispersion data for appropriate immersion liquid using the Pulfrich refractometer. 


\section{B.V. Andriyevsky et al.: Changes of anisotropy of dilatative and ...}

Temperature measurements of the optical path difference $D(T)$ were performed using the laser interferometer of the Jamin type [5]. Two interfering light beams were passing through the sample of the thickness $L_{\mathrm{i}}$ and through the same thickness of air. The measurements of temperature dependence of light intensity were performed in the scanning mode in the heating regime. Since the relative temperature changes of the optical path difference $D_{i j}=L_{i} \cdot\left(n_{j}-1\right)$ can be presented in the form [6],

$$
\frac{\delta D_{i j}}{D_{i j}}=\frac{\delta L_{i}}{L_{i}}+\frac{\delta n_{j}}{\left(n_{j}-1\right)},
$$

we have calculated the relative temperature changes of refractivity $\delta n_{j} /\left(n_{j}-1\right)$ on the basis of relative temperature changes of $\delta D_{i j} / D_{i j}$ and $\delta L_{i} / L_{i}(i, j=g, m, p)$.

The temperature dependence of spontaneous polarization $P_{s}(T)$ of DGN crystal was measured using the known method of pyroelectric current integration [7].

\section{Results and discussion}

Temperature expansion of DGN crystals in the wide temperature range $130 \div 293 \mathrm{~K}$ is characterized by the positive coefficients of linear and volume thermal expansion, $\alpha_{i}=d / d T(\delta L / L)_{i}$ and $\beta=\left(\alpha_{g}+\alpha_{m}+\alpha_{p}\right)$, respectively (Fig. 1). The character of temperature anomalies of $(\delta L / L)_{i}$ and $\mathrm{d} V / V$ corresponds to the continuous type of phase transition (Fig. 1). Linear approximations of the relative change of crystal's dimensions $(\delta L / L)_{i}$ and $\mathrm{d} V / V$ from paraelectric to ferroelectric phase are shown in Fig. 1 (dashed lines). The spontaneous changes of linear dimensions $(\delta L / L)_{i}^{(s)}$ are negative for the direction along $m$ half-axis of optical indicatrix $(i=m)$ and are positive for $p$ - and $g$-half-axes (Fig. 1).

The coefficients of linear and volume spontaneous electrostriction, $Q_{i j}$ and $Q_{V}$ respectively, were calculated on the basis of the following relations [7]:

$(\delta L / L)_{i}^{(s)}=Q_{i j}{ }^{(s)} \cdot P_{j}^{2}=Q_{i j}^{(s)} \cdot P_{s}^{2}$,

$(\delta V / V)^{(s)}=Q_{\mathrm{V}}^{(s)} \cdot P_{s}^{2}$.

It is seen from Fig. 2 that the characteristics of temperature dependences of the square of spontaneous polarization $P_{S}^{2}$ and spontaneous change of the volume $(\delta V / V)^{(s)}$ are different. Similar relation was found for the dependences $P_{S}{ }^{2}(T)$ and $(\delta L / L)_{i}{ }^{(s)}(T)$. These differences are displayed also in the temperature dependence of $Q_{V}(s)$ coefficient as its decrease with approaching $T_{c}$ in ferroelectric phase (Fig. 2).

Taking into account that the spontaneous polarization $P_{S}$ is a primary order parameter in the proper ferroelectric DGN, the non-constancy of $Q_{V}{ }^{(s)}$ coefficient near $T_{c}$ testifies that the $(\delta V / V)^{(s)}$ value is not a "good" secondary order parameter. From the viewpoint of microscopic structure of ferroelectric crystal the spontaneous polarization $P_{S}$ is associated with the certain electrically charged structure elements, whereas the spontaneous deformation $(\delta V / V)^{(s)}$ reflects peculiarities of the integrated microscopic structure. One can also interpret the observed minimal magnitude of the electrostriction coefficients $Q_{V}^{(s)}$ and $Q_{i j}{ }^{(s)}$ in the temperature region close to $T_{c}$ as being caused by the weakened correlation be-

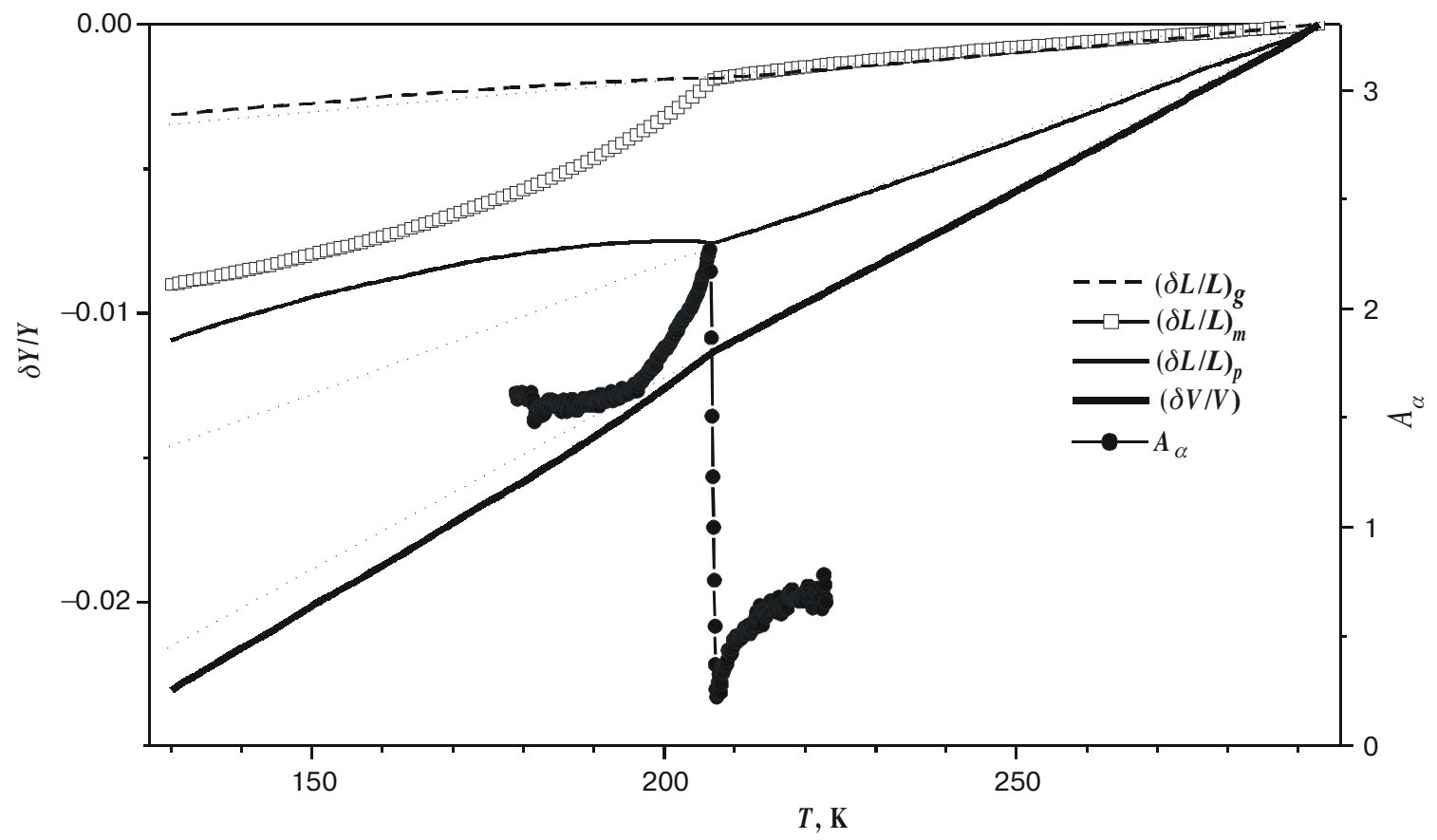

Fig. 1. Temperature dependences of the relative changes of linear dimensions $(\delta L / L)_{i}(i=g, m, p)$, volume $\delta V / V$ and degree of anisotropy $A_{\alpha}$ of DGN crystal in the range of $130-293 \mathrm{~K}$. 


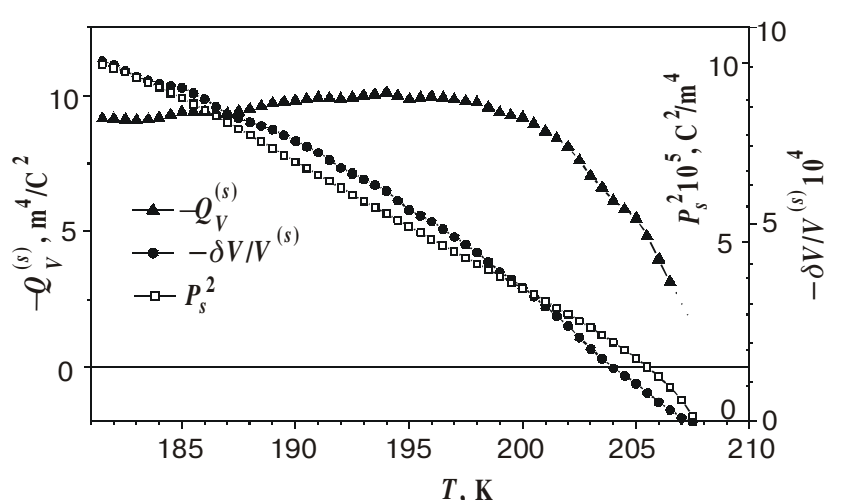

Fig. 2. Temperature dependences of the square of spontaneous polarization $P_{s}^{2}$, relative spontaneous change of volume $(\delta V / V)^{(s)}$ and coefficients of spontaneous electrostriction $Q_{V}^{(s)}$ for DGN crystal.

tween these certain electrically charged elements mentioned above and the remained structure elements of the crystal.

The degree of anisotropy of thermal expansion of DGN crystal can be presented similarly to the definition from [8] using only one value $A_{\alpha}$ which depends on three coefficients of linear expansions $\alpha_{i}$ :

$A_{\alpha}=\frac{\left|\alpha_{g}-\alpha_{m}\right|+\left|\alpha_{m}-\alpha_{p}\right|+\left|\alpha_{p}-\alpha_{g}\right|}{\left|\alpha_{g}+\alpha_{m}+\alpha_{p}\right|}$.

The temperature dependence of $A_{\alpha}(T)$ (Fig. 1) shows more high level of the anisotropy degree $A_{\alpha}$ in ferroelectric phase $\left(T<T_{c}\right)$ than that one in paraelectric phase $\left(T>T_{c}\right)$. Thus, the continuous phase transition in DGN crystal leads to the sharp change of the level of anisotropy degree $A_{\alpha}$ of thermal linear expansion. Besides, the pronounced maximum and minimum of the temperature dependence of $A_{\alpha}(T)$ at $T_{c}$ are observed in the range of 190 $220 \mathrm{~K}$. Similar decrease of the anisotropy degree $A_{\alpha}$ was observed for DMAGaS crystal in the range of paraelectric phase near $T_{c}$ point of continuous phase transition [8]. Therefore one can suppose that the minimum at $T_{c}$ of the temperature dependence of $A_{\alpha}(T)$ in paraelectric phase is a common peculiarity of phase transitions in ferroelectric crystals testifying for the relative disorder of crystal structure. Similar anomalous temperature behavior of $A_{\alpha}(T)$ in the form of maximum at $T_{c}$ is characteristic for ferroelectric phase (Fig. 1). Such pre-transitional anomalous temperature changes of $A_{\alpha}(T)$ in DGN crystal are as deviation from the simple theoretical (classical) constancy of the temperature dependence $A_{\alpha}(T)$ in one structural phase of a crystal with continuous phase transition.

Spectral-interference studies have shown that the dispersion of refractive indices $n(\omega)$ of DGN crystal is normal and monotonous in the photon energy range of $1.77 \div 3.10 \mathrm{eV}$. Analysis of the photon energy dependence of refractive indices $n(\omega)$ in the range under study shows, that this dependence can be satisfactorily described by the single oscillator Sellmeier-like formula

$$
\{n(\omega)-1\}^{-1}=\left(\omega_{0}^{2}-\omega^{2}\right) /\left(k \omega_{0} \Delta \omega_{0}\right)=a-b \omega^{2},
$$

where $\omega_{0}, k$, and $\Delta \omega_{0}$ are resonance photon energies, absorption index $(\hat{n}=n+i k)$, and spectral width of the effective oscillator's band, respectively [8]. The coefficients $a, b, \omega_{0}$, and $k \Delta \omega_{0}$ derived from the dispersion of principal refractive indices of DGN crystals at room temperature are presented in Table 1 . The principal refractive indices of DGN crystal were found to be equal to $n_{p}=1.450, n_{m}=1.530, n_{g}=1.592$ for the wavelength $\lambda=632.8 \mathrm{~nm}(\omega=1,96 \mathrm{eV})$ of He-Ne-laser used in this study for measurement of the temperature dependences of optical path differences $\delta D / D(T)$.

It is seen from Fig. 3 that the principal refractive indices $n_{i}$ increase with decreasing temperature. Clear temperature anomalies of the values $\{\delta n /(n-1)\}_{i}$ observed at $T_{c}=206 \mathrm{~K}$ are characteristic for continuos phase transitions. Relative spontaneous changes of the refractivity $\{\delta n /(n-1)\}_{i}^{(s)}$ are positive, that leads to the positive coefficients of spontaneous quadratic electrooptic effect $G_{i j}{ }^{(s)}$ :

$\{\delta n /(n-1)\}_{i}^{(s)}=G_{i j}^{(s)} \cdot P_{j}^{2}=G_{i j}^{(s)} \cdot P_{s}^{2}$.

Taking into account the magnitudes of $\{\delta n /(n-1)\}_{i}^{(s)}$ at $130 \mathrm{~K}$ (Fig. 3) and the magnitude of spontaneous polarization $P_{S}=1.4 \cdot 10^{-2} \mathrm{C} / \mathrm{m}^{2}$ at $123 \mathrm{~K} \mathrm{[1]} \mathrm{one} \mathrm{can} \mathrm{ob-}$ tain the magnitudes of the corresponding coefficients $G_{i j}(s)$ being in the range of $11 \div 15 \mathrm{~m}^{4} / \mathrm{C}^{2}$.

The temperature changes of electron refractions $(\delta R / R)_{i}$ along three principal directions of optical indicatrix characterizing the electron subsystem of DGN crystal have been calculated by the relation

$$
\frac{\delta R_{i}}{R_{i}}=\frac{\delta V}{V}+\frac{6 n_{i}}{\left(n_{i}+1\right)\left(n_{i}^{2}+2\right)} \frac{\delta n_{i}}{\left(n_{i}-1\right)}
$$

Table 1. The coefficients $a, b, \omega_{0}$, and $k \Delta \omega_{0}$ of the formula (4) for DGN crystal at room temperature.

\begin{tabular}{lllll}
\hline \hline Principal direction of optical indicatrix & $a$ & $b, \mathrm{eV}^{-2}$ & $\omega_{0}, \mathrm{eV}$ & $k \Delta \omega_{0, \mathrm{eV}}$ \\
\hline$g$ & 1.74588 & 0.01513 & 10.74 & 6,15 \\
\hline$m$ & 1.93162 & 0.01182 & 12.78 & 6.62 \\
\hline$p$ & 2.26731 & 0.01178 & 13.87 & 6.12 \\
\hline \hline
\end{tabular}




\section{B.V. Andriyevsky et al.: Changes of anisotropy of dilatative and ...}

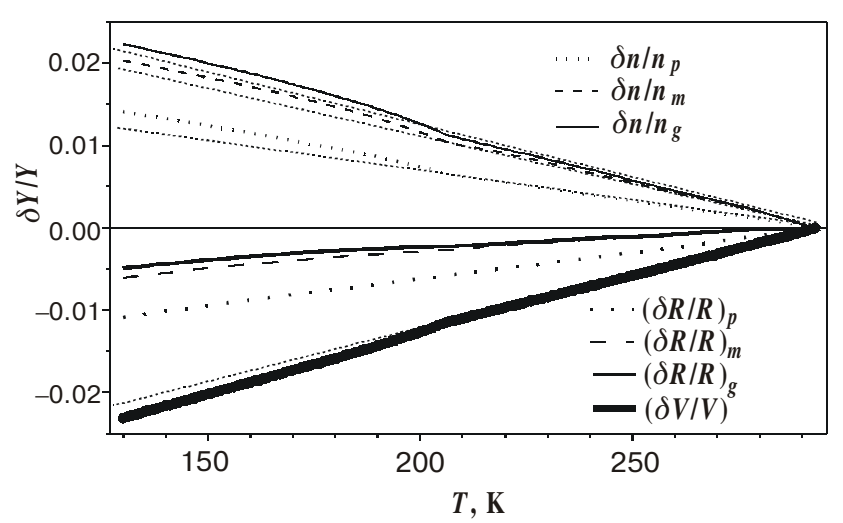

Fig. 3. Temperature dependences of the relative change of refractivity $\delta n_{i} /\left(n_{i}-1\right)(i=p, m, g)$, volume $\delta V / V$ and corresponding refractions $(\delta R / R)_{i}$ for DGN crystal. Thin dashed lines are the linear approximations of corresponding dependences from paraelectric into ferroelectric phase.

derived from the known Lorentz-Lorenz relation (7) for the crystals of cubic structure with relatively strong Lorentz-Lorenz dipole-dipole interaction [5]:

$R_{i}=\frac{\left(n_{i}^{2}-1\right) M}{\left(n_{i}^{2}+2\right) \rho}$.

Here $M$ is the molar mass and $\rho$ is the density of the crystal. The relative temperature change of the nonpolarized refraction $\delta R / R$ was calculated as an arithmetically averaged value of three polarized refractions $\delta R_{i} / R_{i}$. Such the space averaged electron refraction $R$ is the sum of similarly averaged electron polarizabilities for all elements of microscopic crystal structure over one mole of a substance [5], and therefore this value is expected to be rather temperature independent.

It is seen from Fig. 3 that the molar refractions $R_{i}$ calculated by the formula (7) decrease monotonously with decreasing temperature in the range of $295 \div 130 \mathrm{~K}$. Taking into account the definition of the parameter $R$ mentioned above, the observed essential temperature dependence of $\delta R / R$ is rather unexpected. From the other viewpoint, if one supposes the relatively weak dipole-dipole interaction in DGN crystal, then the following relations take place for characteristics $R^{\prime}$ and $\delta R^{\prime} / R^{\prime}[5]$ :

$R^{\prime}=\frac{\left(n^{2}-1\right) M}{3 \rho}$,

$$
\frac{\delta R^{\prime}}{R^{\prime}}=\frac{\delta V}{V}+\frac{2 n}{(n+1)} \frac{\delta n}{(n-1)} .
$$

Our investigations have shown that the value $\delta R^{\prime} / R^{\prime}$ is almost independent on temperature for DGN crystal. Therefore, in the frame of accepted suggestion of temperature independence of electron refraction for DGN crystal in wide temperature range, one can conclude the absence of Lorentz-Lorenz dipole-dipole interaction in this material. But such the explanation is not acceptable because the absence of dipole-dipole interaction in the closed packed substance like DGN crystal is rather impossible. In the frame of suggestion of temperature independence of the additive part of electron refraction $R$ (sum of all non-interacted electron polarizabilities) the obtained temperature coefficient of refraction, $d / d T(\delta R / R)=$ $=(4,0 \div 4,5) \cdot 10^{-5} \mathrm{~K}^{-1}$ (Table 2$)$, calculated on the basis of the relation (7), can be explained by the increase of dipole-dipole interaction in the crystal with decreasing temperature in the wide temperature range $295 \div 130 \mathrm{~K}$. In fact as it is seen from the equations (7) and (8) for the given magnitudes of $n, M$ and $\rho$ the relation $R<R^{\prime}$ takes place. It means that the refraction $R$ can decrease with increasing Lorentz-Lorenz dipole-dipole interaction.

The decrease of Lorentz-Lorenz dipole-dipole interaction with increasing temperature agrees qualitatively with the corresponding decrease of crystal's density. Therefore the ratio of two coefficients $d / d T(\delta R / R)$ and $d / d T(\delta V / V)$ can also serve for the quantitative evaluation of temperature change of Lorentz-Lorenz dipole-dipole interaction in various ferroelectrics:

$K=\frac{d / d T(\delta R / R)}{d / d T(\delta V / V)}$

It is known that the refraction $R$ possessing a dimensionality of volume is approximately equal to the $b$-correction in Van-der-Waals equation, which is equal to the proper volume of molecules for one mole of substance. It is seen from the equation (7) that the ratio of the characteristic volume of electron subsystem of one mole $R$ and the whole volume $V_{M}$ of one mole of substance $(m=M)$ can be presented in the form:

$R / V_{M}=\frac{n^{2}-1}{n^{2}+2}$

So, the ratio $R / V_{M}$ is proportional to the refractive index $n$.

Table 2. Characteristic coefficients of temperature changes of volume $V$, refraction $R$ and refractivity $(n-1)$ of DGN crystal in paraelectric $(207 \div 293 \mathrm{~K})$ and ferroelectric $(130-207 \mathrm{~K})$ phases.

\begin{tabular}{|c|c|c|c|c|}
\hline & & & & $d / d T(\delta R / R)$ \\
\hline & $d / d T(\delta V / V), \mathrm{K}^{-1}$ & $d / d T(\delta R / R), \mathrm{K}^{-1}$ & $d / d T\left(\delta n /(n-1), \mathrm{K}^{-1}\right.$ & $\overline{d / d T(\delta V / V)}$ \\
\hline $207 \div 293 \mathrm{~K}$ & $1.30 \cdot 10^{-4}$ & $4.0 \cdot 10^{-5}$ & $-1.1 \cdot 10^{-4}$ & 0.308 \\
\hline $130 \div 207 \mathrm{~K}$ & $1.47 \cdot 10^{-4}$ & $4.6 \cdot 10^{-5}$ & $-1.3 \cdot 10^{-4}$ & 0.313 \\
\hline
\end{tabular}




\section{B.V. Andriyevsky et al.: Changes of anisotropy of dilatative and ...}

To evaluate the temperature changes of anisotropy of optical indicatrix of DGN crystal we have introduced the single parameter $A_{n-1}$ (degree of refractivity's anisotropy) similarly to the analogous value $A_{\alpha}$ for thermal linear expansion (3):

$A_{n-1}=\frac{\left|n_{g}-n_{m}\right|+\left|n_{m}-n_{p}\right|+\left|n_{p}-n_{g}\right|}{\left|n_{g}+n_{m}+n_{p}-3\right|}$.

It has been stated that the degree of optical anisotropy $A_{n-1}$ of DGN crystal increases with decreasing temperature in the wide range of $295 \div 130 \mathrm{~K}$ (Fig. 4). On the background of monotonous temperature change of the degree of optical anisotropy $A_{n-1}(T)$ (dashed straight line in Fig. 4) the relative decrease of this parameter in the range of $\delta T \approx 30 \mathrm{~K}$ centered by $T_{c}$ temperature is seen in Fig. 4 . This relatively small decrease of $A_{n-1}$ can testifies the approach of valence electron subsystem of DGN crystal to the more isotropic and/or disordered state in the range near $T_{c}$. The characters of temperature anomalies of the relative dilatation $(\mathrm{d} L / L)_{i}$ and refractivity $[\delta n /(n-1)]_{i}$ near $T_{c}$ are similar (Fig. 1,3). Therefore the temperature behaviors of the corresponding degrees of anisotropy $A_{\alpha}$ and $A_{n-1}$ near $T_{c}$ should be similar. Taking into account the form of the temperature dependence $A_{n-1}(T)$ (Fig. 4) one can forecast that the temperature dependence of the absolute value of $d A_{n-1} / d T$ will be similar to the temperature dependence of $A_{\alpha}(T)$ (Fig. 1) discussed earlier.

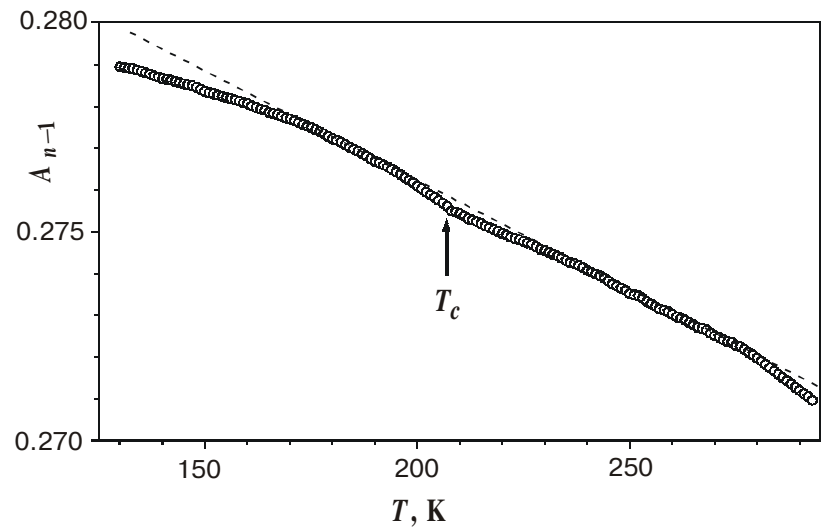

Fig. 4. Temperature dependence of the degree of optical anisotropy $A_{n-1}(T)$ for DGN crystal. The dashed straight line is the background level for the dependence $A_{n-1}(T)$ in the whole temperature range.

\section{Conclusions}

1. Decrease of the spontaneous electrostriction coefficients $Q_{V}{ }^{(s)}$ and $Q_{i j}{ }^{(s)}$ with approaching temperature to the phase transition point $T_{c}$ in ferroelectric phase of DGN crystal can be caused by the weakened correlation between (a) the crystal's structure elements forming the spontaneous polarization $P_{S}$ and (b) the remained structure elements.

2. Relative temperature decrease of the degrees of anisotropy of linear thermal expansion $A_{\alpha}$ and refractivity $A_{n-1}$ of DGN crystal in the range of paraelectric phase close to $T_{c}\left(T_{c} \div T_{c}+10 \mathrm{~K}\right)$ testifies the crystal structure to become to more isotropic and/or relatively disordered.

3 . In the frame of suggestion of temperature independence of the additive part of electron refraction $R$ the observed positive temperature coefficient of refraction $d / d T(\delta R / R)$ can be caused by the increase of the LorentzLorenz dipole-dipole interaction with decreasing temperature in the wide range of $295 \div 130 \mathrm{~K}$ including paraelectric and ferroelectric phases of DGN.

\section{Acknowledgement}

The authors are thankful to Professor Zbigniew Czapla from Wroclaw University, who kindly presented DGN crystal for the investigation.

\section{References}

1. R. Pepinsky, K. Vedam, S. Hoshino and Y. Okaya, Ferroelectricity in Di-Glycine Nitrate $\left(\mathrm{NH}_{2} \mathrm{CH}_{2} \mathrm{COOH}\right)_{2} \cdot \mathrm{HNO}_{3}$ // Phys. Rev. 111(2), pp. 430-432 (1958).

2. S.Sato, Crystal Structure and Phase Transition of (Glycine) ${ }_{2} \mathrm{HNO}_{3} / /$ J. Phys. Soc. Jap. 25(1), pp. 185-201 (1968).

3. V.M. Varikash, N.A. Romanyuk and B.G. Mytsyk, Characterization of indicatrics and piezo-optical properties of diglycine nitrate crystals, Journal of Applied Spectroscopy 37(2), pp. 319-322 (1982).

4. F. Gugenberger, C. Meingast, G. Roth, K. Grube, V. Breit, T. Weber, H. Wühl, S. Uchida and Y. Nakamura, Uniaxial pressure dependence of $T_{\mathrm{c}}$ from high-resolution dilatometry of untwinned $\mathrm{La}_{2-x} \mathrm{Sr}_{x} \mathrm{CuO}_{4}$ single crystals // Phys. Rev. B. 49, pp. 13137-13142 (1994).

5. M. Born, E. Volf, Principal of Optics. Pergamon Press, Oxford/London/Edinburg/New York/Paris/Frankfurt (1968).

6. B. Andriyevsky, O. Myshchyshyn and M. Romanyuk, Interferometric investigation of phase transition in TGS crystal // Physica Status Solidi (b) 203(2), pp.549-555 (1997).

7. M.E. Lines, A.M. Glass, Principles and Application of Ferroelectrics and Related Materials. Clarendon Press, Oxford (1977).

8. D. Podsiadla, Z. Czapla, B. Andriyevsky and O. Myshchyshyn, Dilatometric and optical properties of $\left(\mathrm{CH}_{3}\right)_{2} \mathrm{NH}_{2} \mathrm{Ga}\left(\mathrm{SO}_{4}\right)_{2} \cdot 6 \mathrm{H}_{2} \mathrm{O}$ crystals in paraelectric and ferroelectric phases // Physica Status Solidi (b) 223, pp.729736 (2001). 